\title{
The Impact of Osteopontin Gene Variations on Multiple Sclerosis Development and Progression
}

\author{
Cristoforo Comi, ${ }^{1,2}$ Giuseppe Cappellano, ${ }^{1,3}$ Annalisa Chiocchetti, ${ }^{1,3}$ Elisabetta Orilieri, ${ }^{1,3}$ \\ Sara Buttini, ${ }^{1,2}$ Laura Ghezzi, ${ }^{4}$ Daniela Galimberti, ${ }^{4}$ Franca Guerini, ${ }^{5}$ Nadia Barizzone, ${ }^{1,3}$ \\ Franco Perla, ${ }^{6}$ Maurizio Leone, ${ }^{1,2}$ Sandra D'Alfonso, ${ }^{1,3}$ Domenico Caputo, ${ }^{5}$ Elio Scarpini, ${ }^{4}$ \\ Roberto Cantello, ${ }^{1}$ and Umberto Dianzani ${ }^{1,3}$
}

${ }^{1}$ Interdisciplinary Research Center of Autoimmune Diseases (IRCAD), University of Eastern Piedmont, "Amedeo Avogadro", Novara, Italy

${ }^{2}$ Section of Neurology, Department of Translational Medicine, University of Eastern Piedmont, "Amedeo Avogadro", Novara, Italy

${ }^{3}$ Department of Health Sciences, University of Eastern Piedmont, "Amedeo Avogadro", Novara, Italy

${ }^{4}$ Dino Ferrari Center, The University of Milan, Fondazione Cà Granda, IRCCS Ospedale Haggior Policlinico, Milan, Italy

${ }^{5}$ Multiple Sclerosis Unit, Don C Gnocchi Foundation, IRCCS, S Maria Nascente, Milan, Italy

${ }^{6}$ Department of Neurology, Mondovì Hospital, Mondovì, Italy

Correspondence should be addressed to Cristoforo Comi, comi@med.unipmn.it

Received 15 June 2012; Revised 3 August 2012; Accepted 6 August 2012

Academic Editor: Timothy B. Niewold

Copyright ( $) 2012$ Cristoforo Comi et al. This is an open access article distributed under the Creative Commons Attribution License, which permits unrestricted use, distribution, and reproduction in any medium, provided the original work is properly cited.

\begin{abstract}
Osteopontin is a proinflammatory molecule, modulating TH1 and TH17 responses. Several reports suggest its involvement in multiple sclerosis (MS) pathogenesis. We previously reported that OPN gene variations at the $3^{\prime}$ end are a predisposing factor for MS development and evolution. In this paper, we extended our analysis to a gene variation at the $5^{\prime}$ end on the $-156 \mathrm{G}>\mathrm{GG}$ single nucleotide polymorphism (SNP) and replicated our previous findings at the $3^{\prime}$ end on the $+1239 \mathrm{~A}>\mathrm{C}$ SNP. We found that only +1239 A > C SNP displayed a statistically significant association with MS development, but both $+1239 \mathrm{~A}>\mathrm{C}$ and $-156 \mathrm{G}>\mathrm{GG}$ had an influence on MS progression, since patients homozygous for both +1239A and - 156GG alleles displayed slower progression of disability and slower switch to secondary progression than those carrying $+1239 \mathrm{C}$ and/or $-156 \mathrm{G}$ and those homozygous for +1239 A only. Moreover, patients homozygous for +1239 A also displayed a significantly lower relapse rate than those carrying $+1239 \mathrm{C}$, which is in line with the established role of OPN in MS relapses.
\end{abstract}

\section{Introduction}

Multiple sclerosis (MS) is an inflammatory disease of the central nervous system characterized by an autoimmune response against the myelin sheaths and axons, resulting in progressive neurological dysfunction [1]. Patients with MS display variable clinical course; at onset, approximately $10 \%$ of patients display a primary progressive form (PP), whereas the remainder start out with a relapsing remitting form (RR), and most of them switch to a secondary progressive form (SP) within 10-30 years [2]. Both genetic and environmental factors are involved in the development/progression of MS, and several studies point to a complex inheritance involving interactions between combinations of loci that may influence the immune response $[3,4]$. An increasing bulk of data suggest that osteopontin (OPN) may play a role in the pathogenesis of MS [5]. OPN is a $60 \mathrm{kDa}$-secreted phosphoprotein functioning as a free cytokine in body fluids or as an immobilized extracellular matrix molecule in mineralized tissue [6]. OPN serum levels are increased in several autoimmune diseases and may influence development of these diseases through the OPN immunoregulatory effects enhancing the proinflammatory T helper type 1 (TH1) and TH17 cell responses and inhibiting the $\mathrm{TH} 2$ responses [7].

OPN transcript is abundant in plaques dissected from brains of patients with MS, whereas it is absent in control 
brain tissue; this finding has been confirmed in rat experimental autoimmune encephalomyelitis (EAE) by microarray cDNA analysis of spinal cord tissue [8]. OPN serum levels are higher in relapsing-remitting than in progressive patients, particularly during the relapse $[9,10]$. Chowdhury et al. reported a correlation between cerebrospinal fluid (CSF) OPN levels and disease activity in patients with MS. These levels did not correlate with disability status but were higher in patients with active disease [11].

The human OPN gene (OPN) is located on chromosome $4 \mathrm{q} 22.1$, and single nucleotide polymorphisms (SNPs) are associated with development and/or disease activity of several autoimmune diseases [12-14]. A link between the gene and protein data was suggested by the correlation between some OPN genotypes and OPN serum levels [15]. Four SNPs of the OPN gene $(+282 \mathrm{~T}>\mathrm{C}$ in exon VI: rs $4754 ;+750 \mathrm{C}>\mathrm{T}$ in exon VII: rs11226616; +1083A > G: rs1126772 and +1239A > C: rs9138) in 3' UTR form three haplotype combinations: haplotype A (282T-750C-1083A-1239A), haplotype B (282C-750T-1083A-1239C), and haplotype C (282C750T-1083G-1239C). Carriers of haplotype B and C displayed higher OPN serum levels and higher risk of developing autoimmune diseases than haplotype A homozygotes. Several data suggested that the high OPN levels were due to increased stability of the mRNA coded by haplotype B and C [15]. Regarding MS, we previously found that haplotype A homozygotes displayed about 1.5 lower risk of developing MS and lower OPN serum levels than haplotype B or C carriers. Moreover, clinical analysis showed that haplotype A homozygous patients displayed slower switching from a RR to a SP form and milder disease with slower evolution of disability than patients carrying haplotype B or C [16].

Interindividual differences of OPN expression may be also influenced by variations in the promoter region of $O P N$ that may modulate its transcriptional activity. This role has been suggested for the $-66 \mathrm{~T}>\mathrm{G}$ [17], $-156 \mathrm{G}>\mathrm{GG}$ (rs7687316), and $-443>\mathrm{T}>\mathrm{C}$ [17] SNPs by Giacopelli et al. [18], and we detected a combined effect of $-156 \mathrm{G}>\mathrm{GG}$ and $+1239 \mathrm{~A}>\mathrm{C}$ on risk of systemic lupus erythematosus (SLE) development [14].

According to these findings, the aims of this study were (1) to replicate our previous findings on the $+1239 \mathrm{~A}>\mathrm{C}$ SNP, (2) to investigate the role of the $-156 \mathrm{G}>\mathrm{GG} \mathrm{SNP}$, (3), to assess the impact of these variations on disease evolution.

\section{Materials and Methods}

2.1. Patients. We analyzed 728 Italian patients ( 278 males, 450 females; M/F: 0.62) with MS diagnosed according to the revised McDonald criteria [19] and 1218 randomly selected ethnically and age-matched healthy controls. Patients were consecutive patients enrolled from the Multiple Sclerosis Centers of the "Amedeo Avogadro," University of Eastern Piedmont (Novara), the University of Milan, IRCCS Policlinico Hospital (Milan), the Don C Gnocchi Foundation, IRCCS, S Maria Nascente (Milan), and the "Santa Croce e Carle" Hospital (Cuneo), Italy. Their clinical and demographic features were similar to those of other series [20,21].
Controls were consecutive Italian donors obtained from the transfusion services of the respective hospitals. Patients and controls were unrelated, Caucasian and Italian, matched for age and gender, with no family history of autoimmune diseases in first degree relatives. According to their clinical course, patients were defined as follows [22]:

RR: occurrence of exacerbations, each lasting at least $24 \mathrm{~h}$ and separated by at least one month of inactivity, with full recovery or sequelae $(n=447)$;

PP: steady worsening of symptoms and signs from onset for at least 6 months, whether superimposed with relapses or not, with occasional plateau and temporary minor improvements; $(n=71)$;

SP: initial RR course followed by steady worsening of symptoms and signs for at least 6 months, whether superimposed with relapses or not, with minor remissions, and plateau $(n=210)$.

We performed an analysis of the following outcome measures: time to reach Kurtzke expanded disability status scale [23] (EDSS) score $>3.0$ and time to reach a progressive course, since it was previously shown that OPN SNPs at the $3^{\prime}$ UTR region may influence these measures in MS patients [16]. According to Hawkins and McDonnell [24], disease of patients who, after at least 10 years from onset, had a mild disability, that is, EDSS score $\leq 3.0$, was defined benign MS. Patients who reached secondary progression within 10 years from onset were defined fast progressive. Patients who did not reach the endpoints were excluded.

In RR patients, EDSS score was assessed in remission phase.

The annual relapse rate before treatment was collected in 327 patients with bout onset (RR patients and SP patients) [21]. Only relapses that occurred in the first three years of disease were included in the analysis.

Samples from patients with RR were drawn during remission. All patients gave their informed consent according to the Declaration of Helsinki [25]. The research was approved by the local ethical committee.

2.2. DNA Analysis. Genomic DNA was isolated from peripheral blood mononuclear cells (PBMCs) using standard methods and primers used to evaluate OPN SNPs were the following: $5^{\prime}$-gccgtgaattccacagccatg-3' (OPN F) $5^{\prime}$-ttgaatgtaataagaatttggtgg- $3^{\prime}(\mathrm{OPN} \mathrm{R})($ for $+1239 \mathrm{SNP})$ and $5^{\prime}$ agccctctcaagcagtcatc- $3^{\prime}$ (promo $1 \mathrm{~F}$ ) $5^{\prime}$-cctgtgttggtggaggatgt$3^{\prime}$ (promo 1R) (for $-156 \mathrm{SNP}$ ). PCR products were purified with the EXO/SAP kit (GE, Healthcare, Piscataway, NJ, USA). Sequencing was performed with the ABI PRISMR BigDyeTM Terminator kit (Applied Biosystems, Foster City, CA) on an automatic sequencer (Applied Biosystems 3100 Genetic Analyser) according to the manufacturer's instructions.

2.3. OPN ELISA Assay. Serum OPN concentrations were evaluated in a capture enzyme-linked immunoadsorbent assay (ELISA) according to the protocol provided by the manufacturer (Calbiochem, San Diego, CA). The optical density was measured at $450 \mathrm{~nm}$ with a microplate reader (Bio-Rad, Hercules, CA). The I-smart program was used to 
create a regression curve. All assays were performed in duplicate, and the observer (E.O.) was blinded to the diagnosis.

2.4. Statistical Analysis. Allelic frequencies and outcome measures were compared with the chi-square test with the Yate's correction. Relapse rate was compared with the MannWhitney $U$-test. For the ELISA experiments, the approximation of population distribution to normality was tested by using statistics for kurtosis and symmetry. Results were asymmetrically distributed and consequently presented as median values and percentiles. ELISA data comparisons were performed with the nonparametric Mann-Whitney $U$ test. All $P$ values are 2-tailed and the significance cut-off was $P<0.05$.

\section{Results}

We typed the +1239A $>$ C SNP in 728 patients and 1218 controls and the $-156 \mathrm{G}>\mathrm{GG}$ SNP in 728 patients and 912 controls, not overlapping with the cohorts analyzed in our previous study [16]. The $+1239 \mathrm{~A}>\mathrm{C}$ SNP was analysed because it allows to discriminate between the $\mathrm{A}$ and non-A haplotypes (not carrying versus carrying the +1239 C allele, resp.).

Frequency of +1239 A homozygotes was decreased in MS patients compared to controls ( $46 \%$ versus $52 \%$; $P=0.011$ ), and +1239 A homozygotes displayed 1.27 lower risk of MS than +1239 C carriers (Table 1 ). These findings confirmed our previous results on different groups of 425 patients and 688 healthy controls, showing that carriers of the +1239 A display a slight protection against MS development. Conversely, no statistically significant difference between patients and controls was found for the $-156 \mathrm{G}>\mathrm{GG}$ SNP (Table 2).

Genotypic distribution did not deviate significantly from the Hardy-Weinberg equilibrium in any group (data not shown).

The next step was to assess the impact of these variations on MS evolution, since we previously reported that +1239 A homozygotes displayed slower disease progression and milder disability over time compared to $+1239 \mathrm{C}$ carriers [16]. According to our previous work, disease progression was evaluated by comparing patients switching from RR to SP within 10 years from onset (fast progressive, $n=184$ ) and those remaining RR over 10 years (slow progressive, $n=444$ ) and disease severity was evaluated by comparing patients with an EDSS score $\leq 3.0$ ten years or more after onset (benign MS, $n=194$ ) and those who reached a score $>3.0$ within ten years (non-benign MS, $n=446$ ).

Table 3 shows that the proportion of slow progressive patients was significantly higher in +1239 A homozygotes than in +1239 C carriers ( $80 \%$ versus $63 \%, P<0.0001$ ), whereas no difference was found between -156GG homozygotes and $-156 \mathrm{G}$ carriers $(73 \%$ versus $70 \%, P=0.3)$. Patients homozygous for both +1239 A and $-156 \mathrm{GG}$ showed a significantly higher proportion of slow progressive patients than those carrying $+1239 \mathrm{C}$ and/or $-156 \mathrm{G}$ (95\% versus
TABLE 1: Frequency distribution of $\mathrm{OPN}+1239 \mathrm{~A}>\mathrm{C}$ genotypes in MS patients and healthy controls.

\begin{tabular}{lcc}
\hline Genotype SNP +1239 & $*$ MS $(n=728)$ & ${ }^{\dagger}$ Controls $(n=1218)$ \\
\hline AA & $335(46)$ & $634(52)$ \\
AC & $314(43)$ & $486(40)$ \\
CC & $79(11)$ & $98(8)$ \\
\hline AA & $335(46)$ & $634(52)$ \\
Non-AA & $393(54)$ & $584(48)$ \\
\hline \multicolumn{3}{c}{ OOR $=1.27 P=0.011(95 \%$ CI: $1.05-1.54)$} \\
\hline
\end{tabular}

${ }^{*}$ Multiple sclerosis patients.

${ }^{\dagger}$ number of subjects and proportions are shown in the brackets. Genotypic distribution did not deviate significantly from the Hardy-Weinberg equilibrium in any group (data not shown).

$\ddagger$ Odds ratio (OR), 95\% confidence limits (95\% CI), $\chi^{2}$ test calculated on allelic frequencies, and $P$ values are 2-tailed.

TABLE 2: Frequency distribution of OPN-156G/GG genotypes in MS patients and healthy controls.

\begin{tabular}{lcc}
\hline Genotype SNP -156 & ${ }^{\dagger}$ MS $(n=728)$ & ${ }^{\dagger}$ Controls $(n=912)$ \\
\hline GG/GG & $78(10.7)$ & $112(12.3)$ \\
G/GG & $304(41.8)$ & $384(42.1)$ \\
GG & $346(47.5)$ & $416(45.6)$ \\
\hline \multicolumn{3}{l}{} \\
\hline
\end{tabular}

* Multiple sclerosis patients.

${ }^{\dagger}$ Number of subjects, proportions are shown in the brackets. Genotypic distribution did not deviate significantly from the Hardy-Weinberg equilibrium in any group (data not shown).

$\ddagger$ Odds ratio (OR), 95\% confidence limits (95\% CI), $\chi^{2}$ test calculated on allelic frequencies, and $P$ values are 2-tailed.

$68 \%, P<0.0001)$ and those homozygous for +1239 A only (95\% versus $80 \%, P=0.0094$ ).

Table 3 also shows that the proportion of benign MS patients was significantly higher in $+1239 \mathrm{~A}$ homozygotes than in +1239 C carriers ( $38 \%$ versus $24 \%, P=0.0001)$ and in $-156 \mathrm{GG}$ homozygotes than in $-156 \mathrm{G}$ carriers $(46 \%$ versus $28 \%, P=0.0018$ ). Patients homozygous for both +1239 A and $-156 \mathrm{GG}$ showed a significantly higher proportion of benign MS patients than those carrying $+1239 \mathrm{C}$ and/or $-156 \mathrm{G}$ and those homozygous for $+1239 \mathrm{~A}$ only ( $52 \%$ versus $38 \%, P=0.038$ ).

To further evaluate the clinical impact of $O P N$ variations, we analyzed the relapse rate in bout-onset patients carrying different genotypes. Data were available for 327 patients (157 AA, 170 non-AA). The relapse rate was significantly lower in +1239 A homozygotes than in +1239 C carriers $(0.5 / \mathrm{yr}$ versus $1.3 / \mathrm{yr}, P=0.01$ ), whereas no difference was found between $-156 \mathrm{GG}$ homozygotes and $-156 \mathrm{G}$ carriers $(0.8 / \mathrm{yr}$ versus $1.1 / \mathrm{yr} ; P=0.09$ ) or between subjects carrying both protective genotypes and those carrying at least one predisposing genotype $(0.6 / \mathrm{yr}$ versus $1.2 / \mathrm{yr} ; P=0.06)$ (Table 4 ).

Finally, we explored whether OPN serum levels varied in patients displaying different outcomes. Consistently, we found that benign patients, as well as slow progressive patients, showed significantly lower protein levels compared to nonbenign and fast progressive patients, respectively 
TABLE 3: Frequency distribution of different outcomes in MS patients carrying different OPN genotypes.

\begin{tabular}{|c|c|c|c|c|c|c|}
\hline \multirow{4}{*}{ Outcome } & \multicolumn{6}{|c|}{ Genotypes } \\
\hline & \multicolumn{2}{|c|}{$+1239 \mathrm{~A}>\mathrm{C}$} & \multicolumn{2}{|c|}{$-156 \mathrm{GG}>\mathrm{G}$} & \multicolumn{2}{|c|}{$\begin{array}{l}+1239 \mathrm{~A}>\mathrm{C} \\
-156 \mathrm{G}>\mathrm{GG}\end{array}$} \\
\hline & \multirow{2}{*}{ AA } & \multirow{2}{*}{$\mathrm{C}$} & \multirow{2}{*}{ GG/GG } & \multirow{2}{*}{ G } & AA & $\mathrm{C}$ \\
\hline & & & & & GG/GG & G \\
\hline Fast progressive ${ }^{\mathrm{b}}$ & $57(20)$ & $127(37)$ & $18(27)$ & $166(30)$ & $3(5)$ & $181(32)$ \\
\hline \multirow[t]{2}{*}{ Slow progressive ${ }^{\mathrm{a}}$} & $228^{\mathrm{c}}(80)$ & $216(63)$ & $58(73)$ & $386(70)$ & $57(95)$ & $387(68)$ \\
\hline & \multicolumn{2}{|c|}{$P<0.0001^{\mathrm{d}}$} & \multicolumn{2}{|c|}{$P=0.311$} & \multicolumn{2}{|c|}{$P<0.0001$} \\
\hline Benign $\mathrm{MS}^{\mathrm{a}}$ & $110(38)$ & $84(24)$ & $36(46)$ & $158(28)$ & $35(52)$ & $159(28)$ \\
\hline \multirow[t]{2}{*}{ Non benign $\mathrm{MS}^{\mathrm{b}}$} & $183(62)$ & $263(76)$ & $42(54)$ & $404(72)$ & $32(48)$ & $414(72)$ \\
\hline & \multicolumn{2}{|c|}{$P=0.0001$} & \multicolumn{2}{|c|}{$P=0.0018$} & \multicolumn{2}{|c|}{$P=0.0002$} \\
\hline
\end{tabular}

${ }^{a}$ Patients displaying RR form (slow progressive) or EDSS $\leq 3$ (benign MS) after 10 years from onset.

${ }^{b}$ Number of patients displaying that disease status; proportions are shown in brackets.

Patients displaying either RR course and less than 10 years of followup (29/728) or PP course (71/728) were excluded from the analysis of progression. Patients displaying EDSS $\leq 3.0$ and less than 10 years of followup (88/728) were excluded from the analysis of disability.

${ }^{c}$ Patients switching to SP form (fast progressive) or reaching EDSS > 3 (non-benign MS) within 10 years from onset.

${ }^{\mathrm{d}}$ Statistical analysis was performed by comparing the different outcomes with the $\chi^{2}$ test.

Total number in the analysis of progression: 628 patients: 285 AA; 343 non-AA; 76 GG; 552 non-GG; 60 AAGG; 568 non-AAGG.

Total number in the analysis of course 640 patients: 293 AA, 347 non-AA; 78 GG, 562 non-GG; 67 AAGG, 573 non-AAGG.

TABLE 4: Relapse rate in patients with bout onset displaying different OPN genotypes.

\begin{tabular}{|c|c|c|c|c|c|c|}
\hline \multirow[b]{2}{*}{ Outcome measure } & \multicolumn{6}{|c|}{ Genotype } \\
\hline & $\begin{array}{c}\text { AA } \\
N=153\end{array}$ & $\begin{array}{c}\mathrm{C} \\
N=174\end{array}$ & $\begin{array}{l}\mathrm{GG} / \mathrm{GG} \\
N=33\end{array}$ & $\begin{array}{l}\text { Non-GG } \\
N=294\end{array}$ & $\begin{array}{l}\text { AAGG } \\
N=26\end{array}$ & $\begin{array}{c}\text { CG } \\
N=301\end{array}$ \\
\hline \multirow[t]{2}{*}{ Relapse rate } & $0.5^{\mathrm{a}}(0.2-1)$ & $1.3(0.6-1.7)$ & $0.8(0.4-1.2)$ & $1.1(0.5-1.3)$ & $0.6(0.2-1.3)$ & $1.2(0.5-1.5)$ \\
\hline & \multicolumn{2}{|c|}{$P=0.01^{\mathrm{b}}$} & \multicolumn{2}{|c|}{$P=0.09$} & \multicolumn{2}{|c|}{$P=0.06$} \\
\hline
\end{tabular}

${ }^{a}$ Median values; interquartile ranges are shown in the brackets.

${ }^{\mathrm{b}}$ Mann-Withney $U$ test.

(median value 132 versus $237 \mathrm{ng} / \mathrm{mL}$, interquartile range 94164 versus $189-289 \mathrm{ng} / \mathrm{mL}, P<0.0001$; median value 154 versus $280 \mathrm{ng} / \mathrm{mL}$, interquartile range 100-207 versus 228$341 \mathrm{ng} / \mathrm{mL}, P=<0.0001)$.

\section{Discussion}

This work stems from our previous observation of a protective effect of +1239 A homozygosity at the $3^{\prime} \mathrm{UTR}$ of OPN for MS development and evolution. In our previous paper, this genotype decreased the risk of MS development by 1.56fold [16]. The parallel observation of a combined effect of $+1239 \mathrm{C}$ and $-156 \mathrm{G}$ on risk of (SLE) development [14] prompted this work extending the OPN analysis in MS to $-156 \mathrm{GG}>\mathrm{G}$.

The current data, obtained on a much larger independent population, replicated our previous findings on $+1239 \mathrm{~A}>$ $\mathrm{C}$, showing that the frequency of $+1239 \mathrm{~A}$ homozygotes was decreased in MS patients and that these subjects displayed 1.27 lower risk of MS development than $+1239 \mathrm{C}$ carriers. The same SNPs in the $3^{\prime}$ UTR region of the OPN gene have been studied in 326 Spanish MS patients and 484 controls by other authors. They did not find statistically significant differences between patients and controls, and this apparent discrepancy might be explained by differences in both size and ethnic background of the population under study [26].

By contrast, analysis of $-156 \mathrm{G}>\mathrm{GG}$ SNP did not detect statistically significant differences between patients and controls (OR 0.91, $P=0.25$ ), which indicated that this genetic variation was not associated to MS development. To our knowledge, this is the first paper on this SNP in the MS population.

The most intriguing results were those on the role of these SNPs on the MS course. On the one hand, this study not only confirmed the correlation between $+1293 \mathrm{~A}>\mathrm{C}$ and disease progression, but also strengthened this finding showing that +1239 A homozygotes displayed a lower relapse rate than the other patients. On the other hand, it detected an additional effect of $-156 \mathrm{G}>\mathrm{GG}$ on disease progression since patients homozygous for both $+1239 \mathrm{~A}$ and $-156 \mathrm{GG}$ displayed a milder disease, with slower progression of disability and slower switch to secondary progression, than those carrying $+1239 \mathrm{C}$ and/or $-156 \mathrm{G}$ and those homozygous for +1239 A only. Therefore, $-156 \mathrm{GG}$ homozigosity in the $5^{\prime}$ end of the gene conferred a further protection especially in subjects also carrying the protective genotype at the $3^{\prime}$ end of the gene.

These protective effects might be related to functional outcomes of these $O P N$ variations. In our previous work, in fact, we showed that $+1293 \mathrm{C}$ was associated with a high 
"baseline" production of serum OPN, possibly related to increased stability of the OPN mRNA [15]. Moreover, position -156 seems to fall in a putative binding site for a component of the RUNX family of transcription factors and might influence osteopontin expression [18].

A further point supporting a protective role of AA genotype is provided by the analysis of OPN serum levels in patients displaying different disease outcomes. As a matter of fact, patients showing increased frequency of AA genotype, that is, benign and slow progressive MS patients, displayed lower OPN levels. Moreover, our findings are in line with the work by Kariuki SN et al. who reported that OPN gene variants modulate cytokine levels in SLE [27].

In conclusion, this work confirms that osteopontin and the OPN gene may be involved in MS development and, especially, progression. These observations suggest that this cytokine may be a therapeutic target to counteract MS progression supporting the finding of Steinman et al. showing that anti-OPN antibodies ameliorate the disease course in experimental autoimmune encephalomyelitis [28].

\section{Acknowledgments}

This work was partly supported by Fondazione Cariplo Ricerca (Milan), FISM 2012/R/12 (Genoa), Italian Ministry of Health (Giovani Ricercatori 2007, D.lgs 502/92), and Regione Piemonte (Piattaforme Innovative Project) (Turin).

\section{References}

[1] U. K. Zettl, O. Stüve, and R. Patejdl, "Immune-mediated CNS diseases: a review on nosological classification and clinical features," Autoimmunity Reviews, vol. 11, pp. 167-173, 2012.

[2] B. G. Weinshenker, "The natural history of multiple sclerosis," Neurologic Clinics, vol. 13, no. 1, pp. 119-146, 1995.

[3] M. Comabella and S. J. Khoury, "Immunopathogenesis of multiple sclerosis," Clinical Immunology, vol. 142, pp. 2-8, 2012.

[4] C. Comi, T. Fleetwood, and U. Dianzani, "The role of T cell apoptosis in nervous system autoimmunity," Autoimmunity Reviews. In press.

[5] M. Carecchio and C. Comi, "The role of osteopontin in neurodegenerative diseases," Journal of Alzheimer's Disease, vol. 25, no. 2, pp. 179-185, 2011.

[6] K. X. Wang and D. T. Denhardt, "Osteopontin: role in immune regulation and stress responses," Cytokine and Growth Factor Reviews, vol. 19, no. 5-6, pp. 333-345, 2008.

[7] G. Murugaiyan, A. Mittal, and H. L. Weiner, "Increased osteopontin expression in dendritic cells amplifies IL-17 production by $\mathrm{CD}^{4+} \mathrm{T}$ cells in experimental autoimmune encephalomyelitis and in multiple sclerosis," Journal of Immunology, vol. 181, no. 11, pp. 7480-7488, 2008.

[8] D. Chabas, S. E. Baranzini, D. Mitchell et al., "The influence of the proinflammatory cytokine, osteopontin, on autoimmue demyelinating desease," Science, vol. 294, no. 5547, pp. 1731$1735,2001$.

[9] M. H. J. Vogt, S. Floris, J. Killestein et al., "Osteopontin levels and increased disease activity in relapsing-remitting multiple sclerosis patients," Journal of Neuroimmunology, vol. 155, no. 1-2, pp. 155-160, 2004.
[10] M. Comabella, I. Pericot, R. Goertsches et al., "Plasma osteopontin levels in multiple sclerosis," Journal of Neuroimmunology, vol. 158, no. 1-2, pp. 231-239, 2005.

[11] S. A. Chowdhury, J. Lin, and S. A. Sadiq, "Specificity and correlation with disease activity of cerebrospinal fluid osteopontin levels in patients with multiple sclerosis," Archives of Neurology, vol. 65, no. 2, pp. 232-235, 2008.

[12] A. Chiocchetti, E. Orilieri, G. Cappellano et al., "The osteopontin gene $+1239 \mathrm{~A} / \mathrm{C}$ single nucleotide polymorphism is associated with type 1 diabetes mellitus in the Italian population," International Journal of Immunopathology and Pharmacology, vol. 23, no. 1, pp. 263-269, 2010.

[13] N. Barizzone, M. Marchini, F. Cappiello et al., "Association of osteopontin regulatory polymorphisms with systemic sclerosis," Human Immunology, vol. 72, pp. 930-934, 2011.

[14] S. D’Alfonso, N. Barizzone, M. Giordano et al., "Two singlenucleotide polymorphisms in the $5^{\prime}$ and $3^{\prime}$ ends of the osteopontin gene contribute to susceptibility to systemic lupus erythematosus," Arthritis and Rheumatism, vol. 52, no. 2, pp. 539-547, 2005.

[15] A. Chiocchetti, M. Indelicato, T. Bensi et al., "High levels of osteopontin associated with polymorphisms in its gene are a risk factor for development of autoimmunity/lymphoproliferation," Blood, vol. 103, no. 4, pp. 1376-1382, 2004.

[16] A. Chiocchetti, C. Comi, M. Indelicato et al., "Osteopontin gene haplotypes correlate with multiple sclerosis development and progression," Journal of Neuroimmunology, vol. 163, no. 1-2, pp. 172-178, 2005.

[17] S. Mochida, M. Hashimoto, A. Matsui et al., "Genetic polymorphims in promoter region of osteopontin gene may be a marker reflecting hepatitis activity in chronic hepatitis C patients," Biochemical and Biophysical Research Communications, vol. 313, no. 4, pp. 1079-1085, 2004.

[18] F. Giacopelli, R. Marciano, A. Pistorio et al., "Polymorphisms in the osteopontin promoter affect its transcriptional activity," Physiological Genomics, vol. 20, pp. 87-96, 2005.

[19] C. H. Polman, S. C. Reingold, B. Banwell et al., "Diagnostic criteria for multiple sclerosis: 2010 revisions to the McDonald criteria," Annals of Neurology, vol. 69, no. 2, pp. 292-302, 2011.

[20] M. Trojano, C. Avolio, C. Manzari et al., "Multivariate analysis of predictive factors of multiple sclerosis course with a validated method to assess clinical events," Journal of Neurology Neurosurgery and Psychiatry, vol. 58, no. 3, pp. 300-306, 1995.

[21] L. Castelli, C. Comi, A. Chiocchetti et al., "ICOS gene haplotypes correlate with IL10 secretion and multiple sclerosis evolution," Journal of Neuroimmunology, vol. 186, no. 1-2, pp. 193-198, 2007.

[22] F. D. Lublin and S. C. Reingold, "Defining the clinical course of multiple sclerosis: results of an international survey," Neurology, vol. 46, no. 4, pp. 907-911, 1996.

[23] J. F. Kurtzke, "Rating neurologic impairment in multiple sclerosis: an expanded disability status scale (EDSS)," Neurology, vol. 33, no. 11, pp. 1444-1452, 1983.

[24] S. A. Hawkins and G. V. McDonnell, "Benign multiple sclerosis? Clinical course, long term follow up, and assessment of prognostic factors," Journal of Neurology Neurosurgery and Psychiatry, vol. 67, no. 2, pp. 148-152, 1999.

[25] K. Case, "Protection of patients' rights to privacy," British Medical Journal, vol. 311, no. 7015, p. 1272, 1995.

[26] A. Mas, A. Martínez, V. de las Heras et al., "The 795CT polymorphism in osteopontin gene is not associated with multiple 
sclerosis in Spanish population," Multiple Sclerosis, vol. 13, no. 2, pp. 250-252, 2007.

[27] S. N. Kariuki, J. G. Moore, K. A. Kirou, M. K. Crow, T. O. Utset, and T. B. Niewold, "Age- and gender-specific modulation of serum osteopontin and interferon- $\alpha$ by osteopontin genotype in systemic lupus erythematosus," Genes and Immunity, vol. 10, no. 5, pp. 487-494, 2009.

[28] L. Steinman, S. Youssef, N. Van Venrooij et al., "Response to Comment on: the influence of the proinflammatory cytokine, osteopontin, on autoimmune demyelinating disease," Science, vol. 299, article 1845, 2003. 


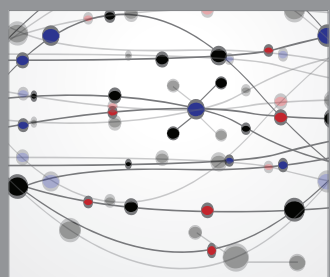

The Scientific World Journal
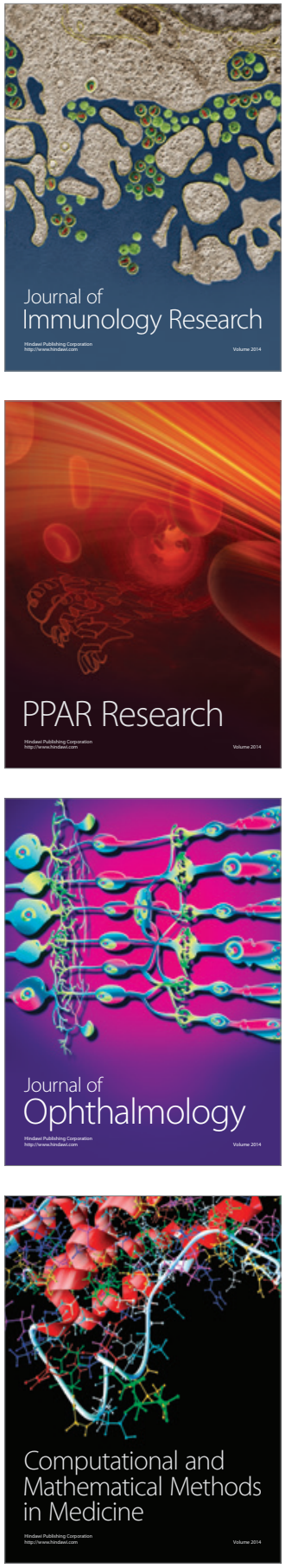

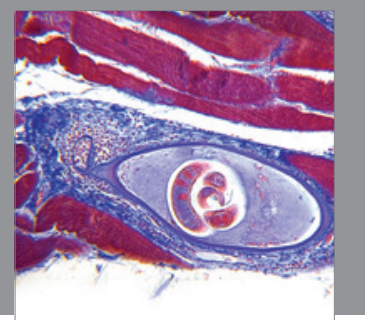

Gastroenterology

Research and Practice
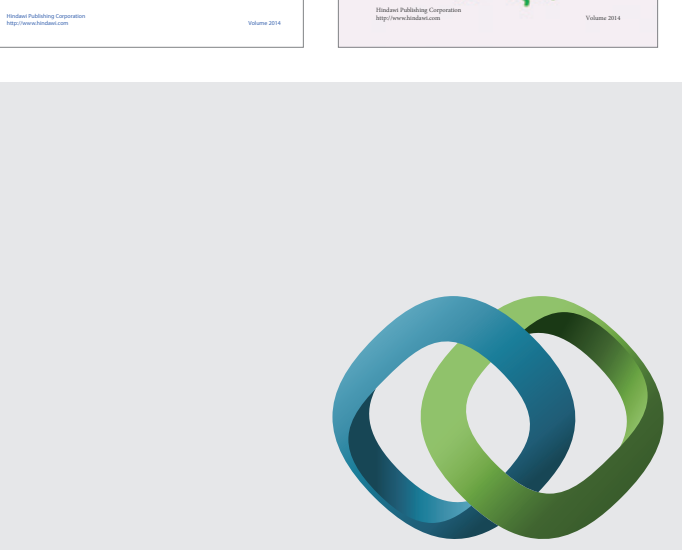

\section{Hindawi}

Submit your manuscripts at

http://www.hindawi.com
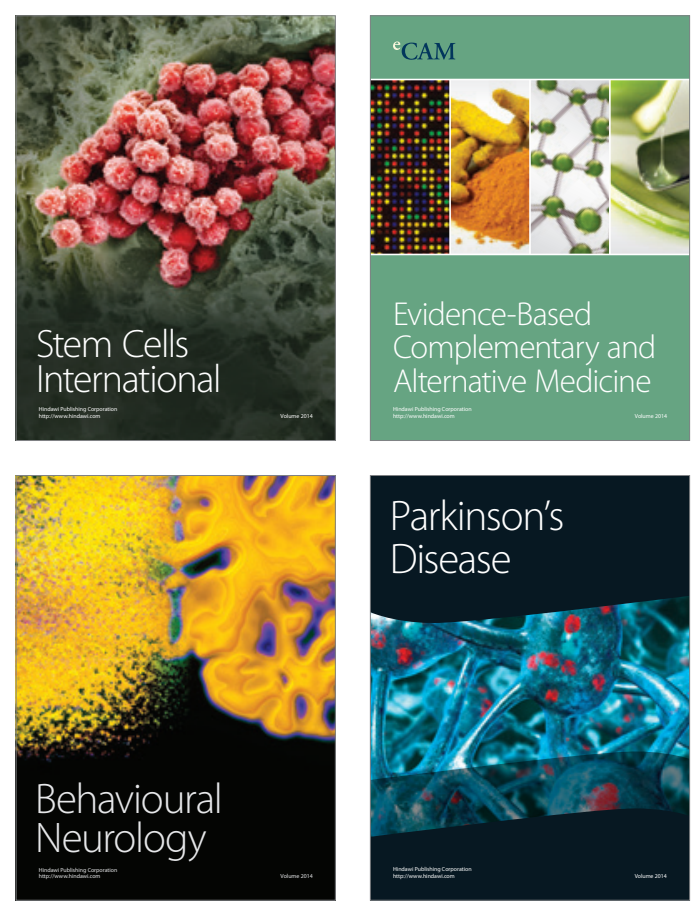

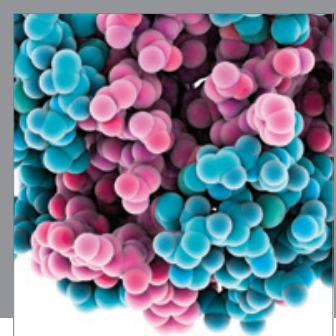

Journal of
Diabetes Research

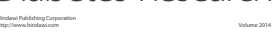

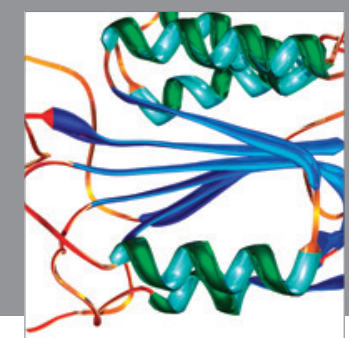

Disease Markers
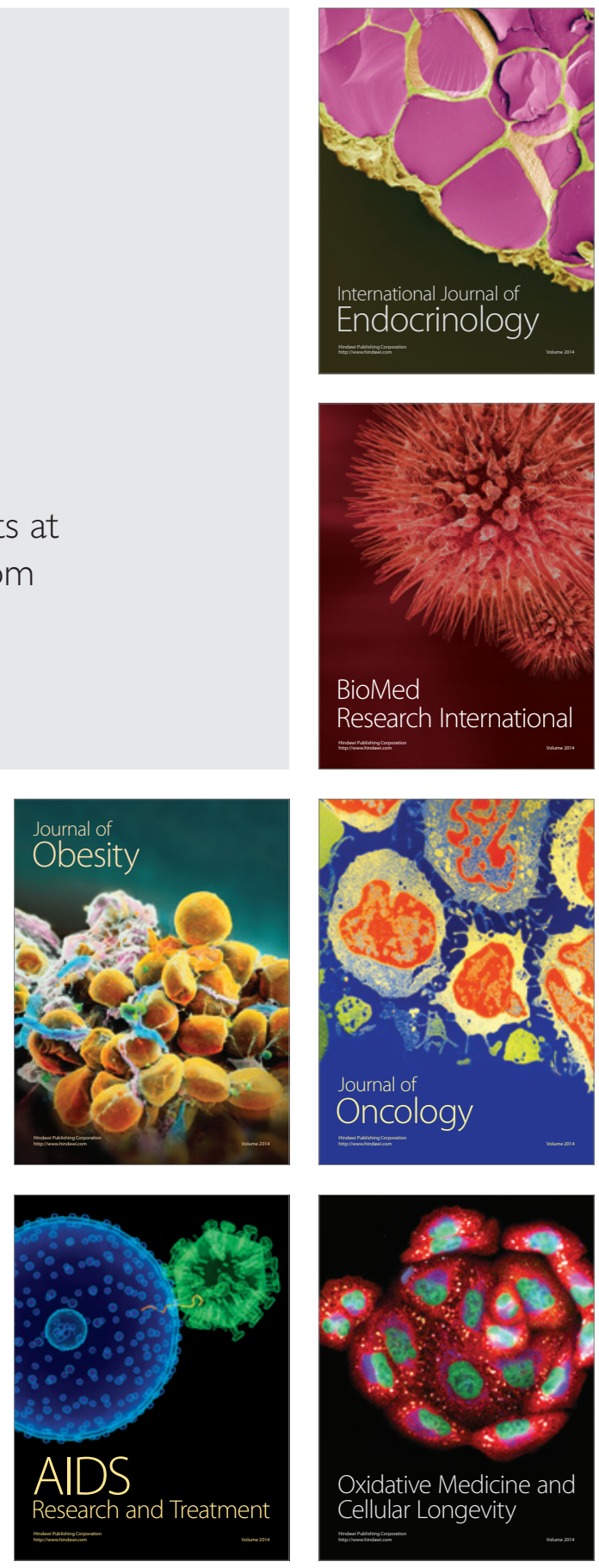